\title{
Demografischer Wandel und Renten: Beschäftigungspotenziale erfolgreich nutzen
}

\author{
Bei der Diskussion über notwendige Maßnahmen zur Bewältigung des demografischen \\ Wandels wird die zukünftige Entwicklung des Arbeitsmarkts oft ignoriert, obwohl Analysen ein \\ enormes ungenutztes Beschäftigungspotenzial vor allem bei Frauen, älteren Menschen und \\ Migranten aufzeigen. Modellrechnungen zeigen, dass eine bessere Nutzung des vorhandenen \\ Beschäftigungspotenzials, höhere Löhnen und eine Fiskalpolitik, die mehr Beschäftigung schafft, \\ dazu beitragen, den demografischen Wandel sinnvoll zu steuern.
}

In den nächsten eineinhalb Jahrzehnten wird sich der Anteil der Rentner an der Bevölkerung stark erhöhen, weil die geburtenstarken Jahrgänge in den Ruhestand gehen, die Mitte der 1950er bis Ende der 1960er Jahre geborenen „Babyboomer“. Berechnungen des Bundesministeriums für Arbeit und Soziales (BMAS) ${ }^{1}$ sowie weitere Studien² zeigen, dass es aufgrund dieser Entwicklung zu einem deutlichen Beitragssatzanstieg in der Rentenversicherung kommt, auch wenn das Rentenniveau abgesenkt wird. Nach aktuellem Rechtsstand ${ }^{3}$ gilt bis 2025 die sogenannte doppelte Haltelinie: Bis dahin soll das Sicherungsniveau ${ }^{4}$ der Rente vor Steuern nicht unter $48 \%$ fallen und der Beitragssatz nicht über $20 \%$ steigen. Sollte das Überschreiten dieser Werte drohen, werden Mittel aus dem Bundes-

(C) Der/die Autor(en) 2020. Open Access: Dieser Artikel wird unter der Creative Commons Namensnennung 4.0 International Lizenz (https:// creativecommons.org/licenses/by/4.0/deed.de) veröffentlicht.

Open Access wird durch die ZBW - Leibniz-Informationszentrum Wirtschaft gefördert.

1 Bundesministerium für Arbeit und Soziales: Gesamtkonzept zur Alterssicherung, Berlin, November 2016, http://www.portal-sozialpolitik.de/uploads/sopo/pdf/2016/2016-11-25_BMAS_Gesamtkonzept_ Alterssicherung.pdf (10.5.2019).

2 Vgl. A. Börsch-Supan, J. Rausch: Die Kosten der doppelten Haltelinie, MEA Discussion paper, Nr. 3, 2018; H. Buslei et al.: Rente mit 67: Der Arbeitsmarkt für Ältere wird entscheidend sein, in: DIW-Wochenbericht, Nr. 16-17, 2019, S. 275-283; M. Werding: Wie variabel ist der demografische Alterungsprozess? Effekte von Geburten und Zuwanderung - Folgen für die soziale Sicherung, Kurzstudie für die Bertelsmann-Stiftung, März 2019.

3 J. Steffen: Übersicht Gesetz über Leistungsverbesserungen und Stabilisierung in der gesetzlichen Rentenversicherung, 2019, http://portalsozialpolitik.de/uploads/sopo/pdf/2018/2018-07-13_RV_LVuStabG_ PS.pdf (3.1.2020).

4 Das Sicherungsniveau vor Steuern bezeichnet das Verhältnis der Standardrente zum Durchschnittsverdienst des jeweiligen Jahres. Die Standardrente ist das Produkt aus dem aktuellen Rentenwert und 45 Entgeltpunkten. Das Sicherungsniveau wird in \% des durchschnittlichen Jahresarbeitsentgeltes aller abhängig Erwerbstätigen angegeben. Von beiden Größen werden die jeweiligen Beiträge zur Sozialversicherung und zur zusätzlichen Altersvorsorge abgezogen, nicht jedoch die Einkommensteuer. haushalt zugeschossen. Vorschläge für das Rentensystem nach 2025 soll die 2018 eingesetzte „Kommission Verlässlicher Generationenvertrag “ 2020 präsentieren.

In vielen Studien werden sehr pessimistische Langfristszenarien bezüglich der Kosten der doppelten Haltelinien erstellt. Die bis 2025 geltenden Regeln werden dabei in die weitere Zukunft fortgeschrieben. ${ }^{5}$ Diese Studien und auch Teile der öffentlichen Diskussion sind durch einen

5 Gemeinschaftsdiagose der Wirtschaftsforschungsinstitute (GD): Deutsche Wirtschaft im Boom - Luft wird dünner, S. 61, 2018, https://www.diw.de/de/diw_01.c.582552.de/themen_nachrichten/gemeinschaftsdiagnose_konjunkturforscher_heben_prognose_leicht an.html (23.4.2018); und A. Börsch-Supan, J. Rausch, a. a. O.

Dr. Rudolf Zwiener ist Referatsleiter am Institut für Makroökonomie und Konjunkturforschung (IMK) der Hans-Böckler-Stiftung.

Dr. Florian Blank ist Referatsleiter im Wirtschaftsund Sozialwissenschaftlichen Institut (WSI) der HansBöckler-Stiftung.

Prof. Dr. Camille Logeay lehrt Volkswirtschaftslehre an der Hochschule für Technik und Wirtschaft in Berlin.

Erik Türk, Mag., ist Referent in der Abteilung Sozialpolitik in der Kammer für Arbeiter und Angestellte in Wien.

Dr. Josef Wöss ist Leiter der Abteilung Sozialpolitik in der Kammer für Arbeiter und Angestellte in Wien. 
eingeschränkten Blick auf die möglichen Handlungsoptionen geprägt: Sie nehmen nur die Rentenversicherung und die dort zur Verfügung stehenden Instrumente wahr. Daher konzentrieren sie sich auf die deutliche Erhöhung der Beitragssätze oder des Zuschusses aus dem Bundeshaushalt, die weitere Absenkung des bereits jetzt sehr niedrigen Leistungsniveaus, ein höheres Renteneintrittsalter oder Kombinationen dieser Maßnahmen. ${ }^{6}$ Häufig bleibt selbst die Option einer Erwerbstätigenversicherung, also die Ausweitung der Versicherungspflicht auf Beamten und Selbständige, unberücksichtigt.

Viele Vorschläge im System der Rentenversicherung spiegeln damit die verkürzte Annahme wider, dass die Relation zwischen Transferempfängern und Erwerbstätigen politisch vor allem mittels Verschiebung der gesetzlichen Altersgrenze zwischen Renten- und Erwerbsalter beeinflusst werden kann. Teils wird noch auf den möglichen Einfluss von Zuwanderung auf die Systeme der sozialen Sicherung verwiesen. ${ }^{7}$ Unser Beitrag weist dagegen auf die gegenwärtig ungenutzten Potenziale des Arbeitsmarktes und den Einfluss einer besseren Arbeitsmarktentwicklung auf die Rentenversicherung hin. Es wird außerdem beispielhaft gezeigt, mit welchen wirtschaftspolitischen Begleitmaßnahmen sich der demografische Wandel für die Rentenversicherung besser bewältigen ließe. Die Rentenversicherung wird damit in einen ökonomischen Kontext gestellt. Dieser Kontext eröffnet Handlungsspielräume zum Umgang mit dem demografischen Wandel, die über die rentenrechtlichen Instrumente weit hinausgehen.

\section{Demografischer Wandel und Sicherungssysteme}

Ein häufig genutzter Indikator für demografische Veränderungen ist der Altenquotient, der die Zahl der älteren Personen (ab 65 Jahre) je 100 Personen im erwerbsfähigen Alter (15 bis 64 Jahre) ausdrückt. Nach den Bevölkerungsvorausschätzungen von Eurostat ${ }^{8}$ wird dieser Altenquotient in Deutschland von $2013^{9}$ bis 2060 um $73 \%$ steigen. Für die Entwicklung der sozialen Sicherungssysteme und ihre Finanzierung sind diese Zahlen aber wenig aussagekräftig. Wichtiger ist das Verhältnis von Leistungsbeziehern und Beitragszahlern bzw. Erwerbstätigen. Diese sogenannte ökonomische Abhängigkeitsquote wird als Quotient der Bezieher von Renten und Arbeitslosenleistungen

6 Zuletzt Deutsche Bundesbank: Staatliche Alterssicherung in Deutschland, Monatsbericht, Oktober 2019, S. 56-79.

7 Teilweise fehlt hier wiederum die Analyse der Erwerbsintegration der Migranten.

8 Eurostat: Bevölkerung am 1. Januar nach Alter, Geschlecht und Art der Vorausberechnung, 2017 (demografische Basis für den EU Ageing Report 2018).

92013 ist das Basisjahr der Langfristprojektionen im Ageing Report 2015 der Europäischen Kommission. an der Zahl der Erwerbstätigen gemessen. ${ }^{10}$ Sie illustriert, wie sich das Verhältnis von wirtschaftlich aktiven und von innen durch Sozialtransfers abhängigen Personen aktuell darstellt und entwickeln wird. Bei einer Bewertung der Folgen des demografischen Wandels geht es schließlich darum, ob Personen durch Erwerbstätigkeit in einem relevanten Ausmaß in die Primärverteilung eingebunden sind, also inren Lebensunterhalt bestreiten und darüber hinaus durch ihre Steuern und Beiträge Sozialtransfers finanzieren können, oder aber wegen Beschäftigungslosigkeit, Erwerbsunfähigkeit oder Alter auf Sozialleistungen angewiesen sind. Es ist offensichtlich, dass das Verhältnis von ökonomisch Aktiven und Sozialleistungsbeziehern stark von der Arbeitsmarktentwicklung abhängt. Entsprechend fließen in Vorausberechnungen der ökonomischen Abhängigkeitsquote nicht nur Annahmen zum demografischen Wandel, sondern auch zum Arbeitsmarkt ein. Werden die vorsichtigen Annahmen der EU-Kommission ${ }^{11}$ zu kommenden Arbeitsmarktentwicklungen in Verbindung mit inrer demografischen Vorausschätzung zugrunde gelegt, steigt im Zeitraum 2013 bis 2060 die ökonomische Abhängigkeitsquote um $41 \%$, also deutlich geringer als der Altenquotient. $^{12}$

Diese Unterscheidung zwischen der rein demografischen und der ökonomischen Betrachtungsweise, also die Berücksichtigung der Arbeitsmarktintegration, ist für die politischen Handlungsoptionen angesichts des demografischen Wandels zentral. Wenn Arbeitskräftepotenziale identifiziert und ausgeschöpft werden, können der durch den demografischen Wandel bedingte tendenzielle Anstieg auch der ökonomischen Abhängigkeitsquote deutlich begrenzt und die Finanzierung der Rentenversicherung erleichtert werden. Das Ausblenden dieser Zusammenhänge führt zu (bestenfalls) unvollständigen politischen Handlungsempfehlungen.

Arbeitsmarktpolitische Handlungsspielräume ergeben sich schon aus den aktuellen Arbeitsmarktzahlen: Bei Frauen, älteren Beschäftigten und Migranten ist die Erwerbsbeteiligung - der Arbeitsmarktstatus wie die Arbeitsstunden deutlich geringer als im Durchschnitt aller Beschäftigten. In manchen Datensätzen sind zudem erhebliche „versteckte" Spielräume vorhanden. So ist im Labour Force Survey, auf den sich auch die EU-Kommission stützt, die Zahl der Erwerbstätigen überhöht und die Zahl der Beschäftigungslosen zu niedrig: In diesem Datensatz sind Personen

$10 \mathrm{Vgl}$. „Yet the real issue is the economic dependency ratio, defined as the unemployed and pensioners as a percentage of the employed." EU Commission: An Agenda for Adequate, Safe and Sustainable Pensions, White Paper, 2012, S. 6.

11 Europäische Kommission: The 2015 Ageing Report, Economic and Budgetary Projections for the 28 EU Member States (2013-2060), European Economy, Nr. 3, Brüssel 2015.

12 E. Türk et al.: Beschäftigungspotentiale nutzen: Die beste Antwort auf die Alterung der Gesellschaft, Wiso Diskurs, Nr. 14, 2018. 
Abbildung 1

Demografischer Wandel

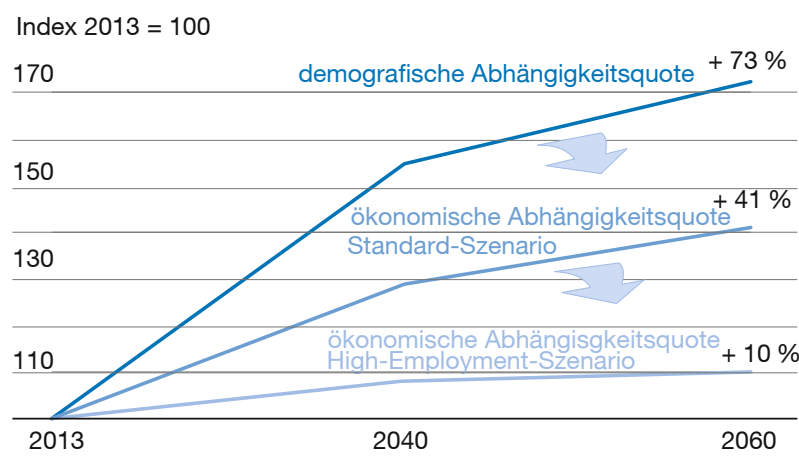

Anmerkungen: Demografische Abhängigkeitsquote = Verhältnis von Personen im Renten- zu Personen im Erwerbsalter: Die Quote lag 2013 bei $32 \%$. Ökonomische Abhängigkeitsquote = Verhältnis von Leistungsbeziehern zu Erwerbstätigen. Die Quote lag 2013 bei $68 \%$.

Quellen: Eurostat 2017; Europäische Kommission: The 2015 Ageing Report; Bundesagentur für Arbeit; eigene Berechnungen.

bereits ab einer Stunde Erwerbstätigkeit pro Woche als erwerbstätig erfasst. Für die Bewertung ökonomischer $A b$ hängigkeitsverhältnisse ist aber nicht das Arbeitsverhältnis per se relevant. Relevant ist Beschäftigung, die Personen zugleich unabhängig von Sozialtransfers macht und die Grundlage für Versicherungsbeiträge und Steuern zur Finanzierung des Sozialstaats bildet.

Zur Berechnung einer möglichst aussagekräftigen ökonomischen Abhängigkeitsquote wurden die Labour-ForceSurvey-Daten in Abbildung 1 angepasst: Ausschließliche Minijobber sollten in dieser Berechnung nicht als erwerbstätig erfasst werden. Eine entsprechende Bereinigung der Daten führt vor allem bei den Frauen - auf die fast zwei Drittel der ausschließlichen Minijobs entfallen - zu einer starken Verminderung der Erwerbstätigenquote im Basisjahr 2013 von $69 \%$ auf $58 \%$. Eine Korrektur, die stärker am ökonomischen Status orientiert und weiter gefasst ist, ist auch bei der Einordnung als "beschäftigungslos" erforderlich. ${ }^{13}$ Die Zahl der Beschäftigungslosen liegt nach diesen Anpassungen im Basisjahr 2013 um $75 \%$ höher als jene der nach dem Labour Force Survey als erwerbslos erfassten Personen: Statt 2,17 Mio. Erwerbslosen in deren Abgrenzung waren es 3,79 Mio. Erwerbslose in der Abgrenzung der Bundesagentur für Arbeit. Die laut Daten des Labour Force Survey vermeintlich heute schon hohe Erwerbsbeteiligung von Männern und Frauen und die scheinbar geringe Erwerbs- oder

13 Diese Korrektur beruht auf der Abgrenzung „Unterbeschäftigung im engeren Sinne" der Bundesagentur für Arbeit: Arbeitsmarkt in Deutschland - Zeitreihen bis 2016; Statistik der Bundesagentur für Arbeit, Berichte: Analyse Arbeitsmarkt, Zeitreihen, Juli 2017, S. 12, S. 54.
Beschäftigungslosigkeit beruhen damit auf sehr problematischen Abgrenzungen. Die Kehrseite der faktisch schlechteren Arbeitsmarktsituation ist ein "verstecktes" höheres ungenutztes und nutzbares Beschäftigungspotenzial von fast 6 Mio. Personen. ${ }^{14}$

Wird von diesen aktuell nicht genutzten Potenzialen ausgegangen und durch eine aktive Wirtschafts- und Arbeitsmarktpolitik darauf hingearbeitet, dass eine Erwerbsintegration erzielt wird, wie sie beispielsweise in Schweden heute schon realisiert ist, könnte der künftige Anstieg der ökonomischen Abhängigkeitsquote gegenüber dem Ausgangsszenario von $41 \%$ auf $10 \%$ reduziert werden. ${ }^{15}$ Das heißt, dass der erwartete Anstieg dieser Quote bei einer wesentlich besseren Erwerbsintegration auf ein Viertel reduziert werden kann. Deutlich wird mit diesen Berechnungen, dass erstens der Anstieg der ökonomischen Abhängigkeitsquote in den kommenden Jahrzehnten geringer sein wird als der Anstieg des Altenquotienten, und zweitens der erwartete Anstieg der ökonomischen Abhängigkeitsquote durch Ausschöpfung des bestehenden Potenzials für eine bessere Erwerbsintegration stark gedämpft werden könnte. Der weit verbreitete Blick allein auf den Anstieg der rein demografischen Relation um $73 \%$ vermittelt ein falsches Bild von einem vermeintlich unabwendbaren Kostenanstieg.

\section{Bessere Wirtschaftspolitik für mehr Beschäftigung}

Im Folgenden wird gezeigt, wie mit einer aktiven Wachstums- und Beschäftigungspolitik, die auf verschiedenen Komponenten der Finanz-, Lohn- und Sozialpolitik beruht, die Entwicklung des deutschen Arbeitsmarktes „in Richtung Schweden" gefördert werden kann, um so (auch) die Wirkung des demografischen Wandels auf die Rentenversicherung zu begrenzen. Ziel ist es zugleich, die soziale Sicherung dauerhaft zu stabilisieren, eine möglichst gute Erwerbsintegration zu gewährleisten, Wachstums- und Einkommensgewinne zu erreichen und Rentner nicht zu belasten. Mit Investitionen in eine bessere öffentliche Infrastruktur und deutlichen Anstrengungen im Bildungsbereich kann das Fundament für höhere Erwerbstätigenquoten und stärkere Produktivitätszuwächse gelegt werden. Politische Maßnahmen in der Arbeitsmarkt-, Wirtschafts- und Sozialpolitik würden teilweise Fehler der Vergangenheit korrigieren (etwa in den Bereichen Rente und Pflege). Dabei kann und sollte die Wirtschaft auch verstärkt ökologisch ausgerichtet werden.

14 Vgl. auch M. Rengers: Ungenutztes Arbeitskräftepotenzial in der stillen Reserve. Ergebnisse für das Jahr 2015, in: Wirtschaft und Statistik, 2016, H. 6, S. 30-51.

15 Vgl. E. Türk et al.: Den demografischen Wandel bewältigen: Die Schlüsselrolle des Arbeitsmarktes, IMK-Report, Nr. 137, 2018, S. 14 ff. $41 \%$ sind im IMK-Report nicht ausgewiesen. Sie ergeben sich für das Standard-Szenario auf Basis der aktuellen Eurostat-Bevölkerungsprognose 2017 
Neben öffentlichen Investitionen nehmen auch höhere Löhne eine wichtige Rolle ein, um diese Wachstumsziele zu erreichen. Letztlich ist es wichtig, dass möglichst viele Personen über verbesserte Beschäftigungsmöglichkeiten und hohe Einkommenssteigerungen am stärkeren Wirtschaftswachstum partizipieren: Wirtschafts- und Verteilungspolitik müssen ineinandergreifen. Es besteht sonst die Gefahr, dass mit zukünftigen Produktivitätssteigerungen zwar die Leistungsfähigkeit der Wirtschaft wächst, diese aber nur einer Minderheit zugutekommt und damit zu wenig dazu beiträgt, die Lasten der Bewältigung des demografischen Wandels fair zu verteilen. Es muss gelingen, neue und bessere Formen der Verteilung des Arbeitsvolumens zu finden und prekäre Formen der Arbeit zurückzudrängen. Den Löhnen kommt eine zentrale Rolle zu. Auf gesamtwirtschaftlicher Ebene sind sie ein wichtiger Kostenfaktor für die Unternehmen, haben einen nennenswerten Einfluss auf die Preisentwicklung und die Staatseinnahmen und sind von eminenter Bedeutung für die Binnennachfrage. Für die Rentenversicherung sind sie von entscheidender Bedeutung für das Beitragsaufkommen und haben gemäß der Rentenformel einen großen Einfluss auf die Rentenanpassungen.

\section{Kombinierte wirtschaftspolitische Maßnahmen}

Ein gesamtwirtschaftliches Modell zeigt für einen Zeitraum von eineinhalb Jahrzehnten, wie mit einem von der Lohnpolitik unterstützten, langfristig ausgerichteten Wachstumsprogramm ein positives Arbeitsmarktszenario erreicht werden kann und wie sich dies auf die Rentenentwicklung auswirkt. Dabei wird ein Politikszenario mit einem Rentenmodell simuliert, das interdependent in ein ökonometrisches gesamtwirtschaftliches Modell integriert ist. Der Vorteil besteht darin, dass es sowohl Wirkungen von der Gesamtwirtschaft auf die Entwicklung der Einnahmen und Ausgaben der gesetzlichen Rentenversicherung als auch die gesamtwirtschaftlichen Effekte von Beitragssatzänderungen, höheren Rentenzugängen und -zahlungen, aber auch Veränderungen der Rentenformel erfasst. ${ }^{16}$ Damit konnte in der Vergangenheit gezeigt werden, dass die Einführung der Riester-Rente und die gleichzeitige Reduzierung des Rentenniveaus zu einer Verlangsamung des Wirtschaftswachstums führten mit dem Ergebnis, dass sich trotz Einsparungen in der Rentenversicherung die öffentliche Finanzlage insgesamt nicht verbesserte. ${ }^{17}$

16 Aufgrund der anderen Abgrenzungen und grundsätzlich verschiedener Modellkonzeptionen können mit dem gesamtwirtschaftlichen Modell ökonomische Abhängigkeitsquoten nicht direkt berechnet werden.

17 Vgl. V. Meinhardt, K. Rietzler, R. Zwiener: Konjunktur und Rentenversicherung - gegenseitige Abhängigkeiten und mögliche Veränderungen durch diskretionäre Maßnahmen, Forschungsprojekt im Auftrag der Deutschen Rentenversicherung Bund, IMK Studies, Nr. 3, 2009.
Grundlage für das Basisszenario des Modells bildet die Bevölkerungsprojektion des Ageing Reports $2018 .{ }^{18}$ Demzufolge ist insbesondere von 2020 bis 2035 mit einem deutlichen Anstieg der Zahl der Rentner und Pensionäre um gut 4 Mio. zu rechnen. Dabei ist die steigende Lebenserwartung berücksichtigt. Eine besondere Herausforderung stellt diese Periode aufgrund des Renteneintritts der Babyboomer dar. Anschließend (bis 2060) werden dann zwar kaum noch Veränderungen bei der Zahl der Rentner und Pensionäre und ein halbwegs stabiles Bevölkerungsniveau von gut 80 Mio. Personen erwartet, doch geht in den EU-Projektionen die Zahl der Erwerbstätigen deutlich zurück, was zu einem Anstieg der ökonomischen Abhängigkeitsquote führt. Dieser Anstieg ist allerdings erheblich schwächer als zuvor in der Phase 2020 bis 2035 bzw. 2040 (vgl. Abbildung 1).

In einem ersten Schritt wird diese demografische Projektion für die Jahre 2020 bis 2035 dem gesamtwirtschaftlichen Rentenmodell für das Basisszenario unterlegt und dabei ermittelt, welche Beitragssatzerhöhung zur Finanzierung der steigenden Zahl von Rentnern notwendig wäre. Dabei wird nach 2025 ein Auslaufen der doppelten Haltelinie und eine Rückkehr zur bisherigen Rentenformel unterstellt. Letztlich müssten gemäß dem Rentenmodell zur Finanzierung der zusätzlichen Rentenausgaben die Beitragssätze zur Rentenversicherung um 3 Prozentpunkte steigen. ${ }^{19}$ Dieser Beitragssatzanstieg und ein Rückgang des Rentenniveaus sind Bestandteil des Basisszenarios.

Deutlich besser sieht die gesamtwirtschaftliche Entwicklung in einem alternativen Politikszenario aus, in dem der Staat mehr für Infrastruktur, Wohnungsbau und Bildung ausgibt (vgl. Tabelle 1), also in Bereichen, in denen in den letzten zwei Jahrzehnten stark gespart wurde. Die zusätzlichen Ausgaben sollten eine ökologische Ausrichtung haben und belaufen sich langfristig in einer Größenordnung von jährlich rund $2 \%$ des BIP. Dabei erfolgt der Ausgabenanstieg in kleinen Schritten, sodass es nicht zu Kapazitätsengpässen kommen kann. ${ }^{20}$ Gleichzeitig fallen in diesem Politikszenario annahmegemäß die jährlichen Lohnsteigerungen um etwa 0,7 Prozentpunkte höher aus, und gleichen so eine lange Phase unterdurchschnittlicher Lohnsteigerungen im ersten Jahrzehnt der Europäischen Währungsunion aus. Das trägt

18 Europäische Kommission: The 2018 Ageing Report, Economic and Budgetary Projections for the 28 EU Member States (2016-2070), Institutional Paper, Nr. 79, Mai 2018.

19 Die für die Basissimulation ermittelte Beitragssatzerhebung stimmt weitgehend mit Berechnungen und Projektionen anderer Simulationsrechnungen überein, vgl. O. Ehrentraut, S. Moog: Zukunft der gesetzlichen Rentenversicherung, Möglichkeiten und Grenzen ausgewählter Reformvorschläge, Study 345 der Forschungsförderung der HBS, 2017, S. 21; A. Börsch-Supan, J. Rausch, a. a. O.; M. Werding, a.a. O.

20 Vgl. auch R. Zwiener: Mehr und besser bezahlte Arbeit statt „Rente mit 70" - Modellsimulation einer erfolgreichen Wachstums- und Beschäftigungspolitik zur Bewältigung des demografischen Wandels, Policy Brief des IMK, Juni 2018. 
zu einem Rückgang der Leistungsbilanzüberschüsse bei und hat einen positiven Effekt auf die Staatsfinanzen. Der Staat profitiert per Saldo von höheren Löhnen aufgrund höherer Steuer- und Beitragseinnahmen, die nur zum Teil für steigende Personal- und Sachausgaben aufgewendet werden müssen. Darüber hinaus wird so die Realeinkommenssituation von Beschäftigten und Rentnern wieder verbessert, die im Basisszenario vor allem durch die Beitragssatzerhöhungen reduziert wird. Zusätzlich wird in der Rentenformel die Riester-Treppe schrittweise herausgenommen; die Rentenanpassungen fallen damit höher aus.

Bei einer solchen positiven Entwicklung ist zu erwarten, dass das Beitragsaufkommen aufgrund der Beschäftigungsgewinne und der höheren Lohnzuwächse, die sich vorübergehend oberhalb der Zielinflationsrate der EZB von knapp $2 \%$ und der mittelfristigen Produktivitätsentwicklung bewegen, deutlich zulegt. Der Anteil am Volkseinkommen, der für Rentner aufgebracht werden muss, nimmt dabei stetig zu. Letztlich kommt das auch den heute Jüngeren zugute, profitieren sie doch dann im Alter selbst von dem vergleichsweise höheren Rentenniveau und den höheren Rentenzahlungen.

In diesem Politikszenario liegt das reale BIP langfristig um gut $5 \%$ und das nominale BIP um gut $12 \%$ höher als im Basisszenario. Das Preisniveau des privaten Verbrauchs steigt um zusätzlich gut $4 \%$. Wachstumstreiber sind vor allem der öffentliche und der private Konsum. Die Beschäftigung nimmt um 4,6\% gegenüber dem Basisszenario zu. Die unterstellten höheren Löhne haben einen positiven Effekt auf die realen Bruttolöhne der Beschäftigten (+ 10,7\% pro Kopf) und die realen Bruttorenten (+ 14,3\% pro Kopf). Letztere profitieren zusätzlich von der Rücknahme der Riester-Treppe in der Rentenformel und der Wirkung des Nachhaltigkeitsfaktors aufgrund der besseren Beschäftigungsentwicklung. Gleichzeitig fallen die Gewinne der Unternehmen und Selbständigen im Vergleich zum Basisszenario um knapp $10 \%$ geringer aus. Letztlich würde damit die gegenüber dem Jahr 2000 gesunkene Lohnquote korrigiert. Die öffentliche Schuldenstandquote bleibt - dank des stärkeren BIP-Wachstums und der höheren Steuereinnahmen - unverändert bei einer gleichzeitig leichten Zunahme der öffentlichen Defizitquote und einer leichten Abnahme der Nachhaltigkeitsrücklage der Rentenversicherung. Mit den im Vergleich zum Basisszenario vorgenommenen Korrekturen an der Rentenformel (schrittweise Rücknahme der Riester-Treppe) wird das Rentenniveau mittelfristig stabilisiert. Die gestiegenen Rentenzahlungen wirken sich vor allem positiv auf den privaten Konsum und das Wirtschaftswachstum aus. Gleichzeitig profitieren die Beschäftigten von der Rückkehr zur paritätischen Finanzierung der Alterssicherung. Die Beiträge zur (nun nicht mehr notwendigen) Riester-Rente mussten sie ohne Arbeitgeberunterstützung aus ihrem verfügbaren Einkommen aufbringen.
Tabelle 1

\section{Gesamtwirtschaftliche Effekte eines expansiven} Politikszenarios

Gesamtwirtschaftliche Effekte

Abweichungen in \% gegenüber Basisszenario

Bruttoinlandsprodukt (BIP), real

BIP, nominal 12,2

Privater Konsum, real 9,3

Anlageinvestitionen, real 6,8

Exporte, real $-3,3$

Importe, real 3,0

Beschäftigte 4,6

Nominallöhne (pro Kopf) $\quad 14,8$

Gewinne $-8,7$

Preisindex privater Konsum 4,1

BIP-Deflator 6,6

Lohnstückkosten 13,7

Bruttorente (pro Kopf), nominal 18,4

nachrichtlich

Rentenausgaben (in Prozentpunkten des BIP) $\quad 0,4$

Staatsausgabenquote (in Prozentpunkten des BIP)

Zunahme der Defizitquote (in Prozentpunkten des BIP) $\quad 0,8$

Lohnquote (in Prozentpunkten des Volkseinkommens) $\quad 5,9$

Schuldenstandquote (in Prozentpunkten des BIP) $\quad 0,0$

Nachhaltigkeitsrücklage (absolut, in Mrd. Euro)

$-3,2$

1 Gesamtwirtschaftliche Effekte von Beitragssatzerhöhungen, expansiver Finanz- und Lohnpolitik, Abschaffung der Riester-Treppe und zusätzliche öffentliche Ausgaben für Infrastruktur, Bildung, Wohnungsbau im Umfang von $2 \%$ des BIP. Die Simulationen wurden für 1994 bis 2007 durchgeführt. Der bis 2035 erwartete Anstieg der Zahl der Rentner (und Beamten) um gut 4 Mio. Personen und des Beitragssatzes um 3 Prozentpunkte wurde übertragen. Ausgewiesen sind die finanzpolitischen Impulse im 14. Jahr der Simulation, nachdem sie schrittweise erhöht wurden. Die entsprechenden Variablen werden zudem von der induzierten wirtschaftlichen Entwicklung beeinflusst.

Quelle: Simulationen mit dem makroökonometrischen Rentenmodell des Instituts für Makroökonomie und Konjunkturforschung IMK.

\section{Folgen für die Alterssicherung}

Die Szenarien-Rechnung zeigt, dass der Arbeitsmarkt (Erwerbsintegration, Lohnhöhe) die zentrale Stellschraube für eine erfolgreiche Bewältigung des demografischen Wandels ist. Der Arbeitsmarkt wiederum ist durch politische Maßnahmen beeinflussbar, die gut bezahlte Beschäftigung fördern. Höhere Löhne haben nicht nur Vorteile für die Beschäftigten, sondern verbessern auch die reale Kaufkraft der Rentner und die Haushaltslage des Staats und tragen - durch höhere Importe - zum außenwirtschaftlichen Gleichgewicht bei. Bestimmend für den zukünftigen Finanzierungsbedarf für Renten sind letztlich zwei Faktoren: Die Entwicklung der 
Zahl der Rentner in Relation zur Zahl der sozialversicherungspflichtig Beschäftigten und die Entwicklung der Renten relativ zu den Arbeitseinkommen.

Von 2020 bis 2035 sind wegen der erwarteten starken demografischen Veränderungen höhere Rentenversicherungsbeiträge und/oder Steuerzuschüsse unausweichlich. Dies gilt insbesondere, wenn die Leistungskürzungen der Vergangenheit wieder ausgeglichen werden sollen, zumindest aber das sonst ab 2025 wieder sinkende Sicherungsniveau dauerhaft stabilisiert werden soll. In den darauffolgenden Jahrzehnten können höhere Erwerbstätigen- bzw. Beschäftigtenquoten den weiteren Anstieg der ökonomischen Abhängigkeitsquote erheblich eindämmen und damit helfen, den demografisch bedingten Ausgabenanstieg mit deutlich geringeren Beitragssatzanstiegen und/oder Steuerzuschüssen zu bewältigen. Die zusätzlich Erwerbstätigen erlangen zudem mit ihren Beiträgen eigene bzw. höhere Ansprüche an die Rentenversicherung, die sie im Alter besser absichern. Es ist offensichtlich, dass es hierbei um sichere Arbeit zu guten Löhnen gehen muss mit Bedingungen, die eine lange Erwerbsintegration unterstützen.

Neben der Finanzierung der Rentenversicherung im demografischem Wandel trägt die vorgeschlagene Strategie auch dazu bei, weitere Probleme zu lösen: Ausbildungsdefizite, mangelnde Vereinbarkeit von Beruf und Familie, schlechte Arbeitsmarktintegration von Migranten und das Fehlen altersgerechter Arbeitsplätze sind Probleme, deren Lösung dabei hilft, die gesellschaftliche und ökonomische Basis sozialer Sicherung weiterzuentwickeln. Ausreichend hohe Erwerbseinkommen und eine gute spätere Alterssicherung werden beispielsweise viele Frauen nur erreichen, wenn es genügend Kinderbetreuungsplätze gibt, die Sorgearbeit fair zwischen den Geschlechtern verteilt wird und sie deshalb nicht mehr im Regelfall halbtags oder gar nur in Minijobs arbeiten müssen. Gute Arbeitsplätze ermöglichen es den Menschen, angemessene eigene Ansprüche gegenüber der Sozialversicherung aufzubauen.

Jenseits des Fokus auf Erwerbsintegration bleibt festzuhalten, dass in einer wachsenden Wirtschaft weitere Verteilungsspielräume vorhanden sind. Durch Produktivitätssteigerungen sind Güter und Dienstleistungen mit weniger Arbeitsstunden herzustellen, wodurch der demografische Wandel leichter bewältigt werden kann. Es muss allerdings sichergestellt werden, dass diese Produktivitätsfortschritte den Beschäftigten zugutekommen und teilweise, über höhere Beiträge und Steuern, auch den Rentnern und weiteren Sozialleistungsempfängern. Produktivitätszuwächse allein entlasten die Politik nicht; sie müssen aktiv verteilt werden.

In der Rentenanpassungsformel wirkt ein - relativer - Anstieg der Zahl der sozialversicherungspflichtig Beschäftig- ten über den Nachhaltigkeitsfaktor rentensteigernd. Ein höheres Arbeitsvolumen der Teilzeitbeschäftigten steigert als Lohnerhöhung pro Kopf ebenfalls die Renten, auch wenn der Stundenlohn unverändert bleibt. Mit diesen Konstruktionsmerkmalen kommt ein höheres Beitragsvolumen, das auf einem im Vergleich zum Basisszenario höheren Arbeitsvolumen - mehr Beschäftigte und/oder mehr Stunden pro Beschäftigte - beruht, auch allen Rentnern zugute und steht daher nur teilweise bzw. in geringerem Ausmaß zur Finanzierung zusätzlicher Rentner zur Verfügung. Das ist aber kein systemischer Zusammenhang, sondern Konsequenz der aktuellen Ausgestaltung der Rentenanpassungsformel, die - falls sich das als sinnvoll erweist - auch geändert werden kann. Von höheren Stundenlöhnen profitieren Beschäftigte und Rentner gleichermaßen, selbst wenn sich dabei das Rentenniveau kaum verändert.

\section{Weitere wichtige Reformen}

Mit dem Fokus auf der grundsätzlichen Bedeutung der Arbeitsmarktentwicklung für die Bewältigung des demografischen Wandels soll nicht infrage gestellt werden, dass es einer Reihe weiterer wichtiger Reformen zur Stärkung der gesetzlichen Rentenversicherung bedarf. Dazu gehört schon kurzfristig ein höherer staatlicher Zuschuss von 25 Mrd. bis 50 Mrd. Euro, um die bestehende Fehlfinanzierung bei den Maßnahmen des sozialen Ausgleichs in der Rentenversicherung zu reduzieren, ${ }^{21}$ eine Abschaffung der Sozialversicherungsfreiheit der Entgeltumwandlung, um die Finanzierung und das Rentenniveau zu verbessern, und eine bessere Absicherung bei Erwerbsunfähigkeit. ${ }^{22}$ Erwähnt wurde schon die sachlich gebotene Rücknahme der Riester-Treppe aus der Rentenformel. ${ }^{23}$

Deutlich gestärkt würde die Rentenversicherung durch den schrittweisen Ausbau zu einer Erwerbstätigenversicherung nach österreichischem Vorbild, in die entweder schrittweise alle Selbständigen und Beamten unter Wahrung ihrer bisherigen Ansprüche ${ }^{24}$ oder nur neue Selbständige und Beamten einbezogen würden. ${ }^{25}$ Der zusätzliche Finanzierungs-

21 Vgl. V. Meinhardt: Versicherungsfremde Leistungen der Sozialversicherung, IMK Studies, Nr. 60, 2018.

22 Die bisherigen Verbesserungen bei der Absicherung der Erwerbsunfähigkeit gehen in die richtige Richtung.

23 Vgl. H. Joebges et al.: Auf dem Weg in die Altersarmut - Bilanz der Einführung der kapitalgedeckten Riester-Rente, IMK Report, Nr. 73, 2012.

24 F. Blank et al.: Österreichs Alterssicherung: Vorbild für Deutschland?, in: Wirtschaftsdienst, 96. Jg. (2016), H. 4, S. 279-286.

25 M. Werding: Alterssicherung, Arbeitsmarktdynamik und neue Reformen: Wie das Rentensystem, stabilisiert werden kann, 2013; H. Buslei et al.: Ausweitung der gesetzlichen Rentenversicherung auf Selbständige: merkliche Effekte auch in der mittleren Frist, in: DIW Wochenbericht, Nr. 30, 2016. In beiden Studien sind relativ lang wirkende und signifikante Transitionsgewinne zu sehen. $\mathrm{H}$. Buslei et al. simulieren bis 2040 bei einer Einführung 2015 und M. Werding bis 2060 bei einer Reform im Jahr 2013. 
bedarf fiele für eine Übergangszeit bei den Gebietskörperschaften an. Gerade in der nun direkt vor uns liegenden Phase mit der Verrentung der Babyboom-Generation wäre das eine hilfreiche Ausweitung der Finanzierungsbasis. Insgesamt würde die Transition wie ein positiver langanhaltender Arbeitsmarktschock für die Rentenversicherung wirken: In der Zeit der Überführung von Selbständigen und Beamten würden die neuen Pflichtversicherten für Entlastung durch zusätzliche Beitragszahlungen sorgen, denen erst einige Jahrzehnte später zusätzliche Rentenansprüche gegenüberständen. Durch eine sukzessive Angleichung der (deutlich) großzügigeren Regelung für Beamtenpensionen an jene der Rentenversicherung plus Zusatzversorgung im öffentlichen Dienst resultieren auch langfristig wirksame Entlastungseffekte für die Gebietskörperschaften, die auch für eine Erhöhung der Rentenniveaus in der Rentenversicherung genutzt werden können. Letztlich würde die starke Zunahme der sozialversicherungspflichtig Beschäftigten bzw. Beitragszahler die steigende Zahl an Rentnern für eine lange Zeit nennenswert finanzieren. Schon seit 2005 steigt die Zahl der sozialversicherungspflichtig Beschäftigten deutlich stärker als die Zahl der Erwerbstätigen insgesamt (und die der Rentner), während die Zahl der Selbständigen und Beamten - zuletzt auch die der Minijobs - zurückgegangen ist. Hält dieser Trend an, dann stärkt allein dies schon die zukünftige Finanzbasis der Rentenversicherung.

Häufig wird eine weitere Heraufsetzung des Renteneintrittsalters als zwingender Bestandteil der anstehenden Rentenreformen betrachtet. ${ }^{26}$ Dabei dauert es noch bis 2029, bis die neue Regelaltersgrenze von 67 Jahren für alle verbindlich ist. Bevor sich die Politik mit neuen Regelaltersgrenzen beschäftigt, muss sie allerdings dafür Sorge tragen, dass ältere Beschäftigte auch tatsächlich in der Lage sind, länger zu arbeiten. Dazu müssen die Unternehmen mehr Arbeitsplätze altersgerecht gestalten und bereit sein, auch ältere Arbeitslose einzustellen. Ansonsten ist eine Veränderung der Regelaltersgrenze nichts anderes als eine Rentenkürzung vor allem für die Berufsgruppen mit in der Regel unterdurchschnittlichen Einkommen und häufig auch einer kürzeren Lebenserwartung. Aufgrund der drastischen Rentenkürzungen der Vergangenheit geht es jetzt darum, das Rentenniveau wieder anzuheben und nicht weiter abzu-

26 Zuletzt forderte die Deutsche Bundesbank langfristig eine Anhebung auf über 69 Jahre; vgl. Deutsche Bundesbank, a. a. O. senken. Auch erscheint es sinnvoll, prioritär die noch ungenutzten Beschäftigungspotenziale innerhalb der bestehenden Grenzen konsequent auszuschöpfen, statt zuerst die Grenzen weiter nach oben zu verschieben.

\section{Fazit}

Für die Frage nach den Folgen des demografischen Wandels, aber auch nach den Handlungsmöglichkeiten bezüglich des Rentensystems ergibt sich damit als Fazit, dass neben den umstrittenen rentenrechtlichen Möglichkeiten - höhere Beitragssätze, höheres Renteneintrittsalter, niedrigeres Sicherungsniveau - der Arbeitsmarkt einen starken Hebel darstellt, um die künftigen Herausforderungen zu bewältigen. Ein starker Arbeitsmarkt sollte durch eine gute Arbeitsmarkt-, Wirtschafts- und Lohnpolitik erreicht bzw. begleitet werden. Ziel ist die Ausweitung des Beitragsvolumens zur Finanzierung angemessener, stabiler Leistungen bei einer steigenden Zahl von Leistungsbeziehern in der Rentenversicherung. Dies umso mehr, wenn zudem Leistungsverbesserungen zu großen Teilen aus Beiträgen finanziert werden sollen.

Mit der hier konzipierten Wachstums- und Beschäftigungspolitik lässt sich innerhalb von eineinhalb Jahrzehnten das Wachstum um zusätzlich gut 5 Prozentpunkte erhöhen und die Zahl der Erwerbstätigen gegenüber den Status quo um fast 2 Mio. Personen anheben. Damit könnte der Weg hin zu schwedischen Erwerbstätigenquoten zumindest zu einem nennenswerten Teil beschritten werden.

Am Beispiel Österreich lässt sich zudem exemplarisch zeigen, dass eine umlagefinanzierte Erwerbstätigenversicherung mit einem erheblich höheren Leistungsniveau auch langfristig nachhaltig aufgestellt ist. ${ }^{27}$ Mit einem schrittweisen Übergang zu einer Erwerbstätigenversicherung in Deutschland gäbe es auch hierzulande für lange Zeit einen Zuwachs an Beitragszahlern. Das Rentenniveau ließe sich so leichter stabilisieren. Das Beispiel Österreich zeigt sehr deutlich, dass die Behauptungen von „Unfinanzierbarkeit“ oder "Gefährdung des Wirtschaftsstandorts" bei höheren Rentenleistungen empirisch nicht belegt sind.

27 F. Blank et al.: Ist das österreichische Rentensystem nachhaltig?, in: Wirtschaftsdienst, 98. Jg. (2018), H. 3, S. 193-199.

Title: Demographic Change and Pensions: More Employment and Higher Wages are Crucial

Abstract: When discussing the necessary measures for coping with demographic change, the future development of the labour market is often ignored despite the fact that analysis shows enormous untapped employment potential, especially among women, older people and migrants. With better usage of existing employment potential, higher wages and a fiscal policy that ensures increased employment, model calculations show that demographic change can be managed sensibly.

JEL Classification: H55, J11, J26, J32 